\section{Acute medical illness and disability in the elderly}

\author{
Lorenzo Palleschi, ${ }^{1}$ Flavia Galdi, ${ }^{2}$ \\ Claudio Pedone ${ }^{2}$
}

${ }^{1}$ Unit of Geriatrics, A.O. San Giovanni

Addolorata, Roma; ${ }^{2}$ Unit of Geriatrics, Università Campus Bio-Medico, Roma, Italy

\begin{abstract}
Acute illness and hospitalization are important events in the trajectory leading to disability in elderly people. Approximately $30-40 \%$ of elderly patients are discharged from hospitals with new disabilities. This rate may increase to $50 \%$ in people aged 85 and older. Hospitalization for an acute medical illness is a stressful and potential dangerous event for older persons that often leads to clinical complications, such as functional decline, prolonged length of stay, readmission, falls and mortality. Very old frail patients and those with preadmission functional limitation are at higher risk of complications. A new model of hospital care focused on functional status, including assessment on admission and throughout the hospital stay, promoting physical activity with early ambulation and planning for discharge home, is needed to reduce the incidence of hospitalization-associated disability and to increase the likelihood of going home reducing hospital length of stay.
\end{abstract}

\section{Introduction}

Acute illness and hospitalization are important events in the trajectory leading to disability in elderly people. Hospitalizationassociated disability (HAD) is recognized as a Geriatric Syndrome and is described as a new or additional disability in activities of daily living (ADL) at hospital discharge compared to the baseline level of about 15 days before hospitalization (retrospectively measured at admission). ${ }^{1}$ It is calculated that $35 \%$ of elderly patients decline in ADL function between baseline and discharge. This rate may increase to $50 \%$ in people aged 85 and older. ${ }^{2}$ When elderly patients experience HAD they are at increased risk of post-discharge negative outcomes, including death, nursing home placement, short-term rehospitalization and prolonged disability. ${ }^{3,4}$ In fact, one year following discharge, fewer than half of older adults have recovered to their pre-illness levels of functioning and rates of nursing home placement and death are high. ${ }^{5}$

Functional trajectory relating to hospitalization is composed by two different and following segments: i) a pre-hospital segment, during which frail elderly can experience functional decline caused by the effect of the acute illness; ${ }^{6}$ ii) an in-hospital segment during which elderly patients can undergo to further functional loss due to multiple iatrogenic factors.?

Recent studies have suggested that a significant part of the functional decline occurs in the few days before admission to hospital and just $1-7 \%$ of patients undergo to functional decline during hospitalization. ${ }^{8}$

\section{Risk factors}

As a geriatric syndrome, HAD can be explained by many causes, reported in the following paragraphs.

\section{Pre-hospital risk factors}

Age is the most important risk factor: more than $50 \%$ of patients older than 85 are discharged from hospital with a new ADL disability; ${ }^{2}$

- Cognitive impairment ${ }^{6}$

- Depression;

- Limited social support;

- Lower pre-admission functional level (before the acute illness); ${ }^{6}$

- Severity of acute illness; ${ }^{9}$

- Comorbidity. ${ }^{1,9}$

It was found that older age, a lower level of physical function at pre-admission, dementia and only acute respiratory diseases among the comorbidities were independently associated with experiencing pre-hospital functional decline. ${ }^{6}$ In particular, approximately $36 \%$ of elderly patients with community acquired pneumonia had functional decline during hospitalization. ${ }^{10}$ It was found that the severity of illness is a strong determinant of pre-hospital functional decline indicating a baseline functional vulnerability to the acute diseases (frailty); in the same study, this pre-hospital vulnerability was the main determinant of overall HAD (decline between pre-morbid baseline and discharge) with a superior statistical strength than illness severity and advanced age. ${ }^{9}$

\section{In-hospital risk factors}

Hospital processes can play an important role both in inhibiting recovery of functional loss occurred before admission and in worsening functional decline:
Correspondence: Lorenzo Palleschi, Unit of Geriatrics, A.O. San Giovanni Addolorata, Roma, Italy. E-mail: 1palleschi@gmail.com

Key words: Frailty; hospitalization; disability.

Received for publication: 15 May 2018 Revision received: 4 September 2018. Accepted for publication: 17 September 2018.

This work is licensed under a Creative Commons Attribution-NonCommercial 4.0 International License (CC BY-NC 4.0).

(C) Copyright L. Palleschi et al., 2018

Licensee PAGEPress, Italy

Geriatric Care 2018; 4:7561

doi:10.4081/gc.2018.7561

Immobility: a study demonstrates that $83 \%$ of time is spent in bed and only $4 \%$ of patients stand or walk. ${ }^{11}$ Physiologically, immobility leads to decreased muscle strength and aerobic capacity, increased bone loss and the development of vasomotor instability. Clinically, immobility is a risk factor for deconditioning, delirium, aspiration events, pressure ulcers and falls; ${ }^{12}$

Polypharmacy: ${ }^{13}$ medications are frequently associated with adverse outcomes, such as falls; ${ }^{14}$

- Iatrogenic complications including adverse drug reactions and infections;

- Hospital environment: it is not always safe, and discourages mobility, exacerbates disorientation, disrupts sleep, leads to social isolation, and increases the likelihood of falls; ;,15,16

Deterioration of nutritional status: ${ }^{1}$ is often a result of prolonged periods of orders restricting oral food intake, a diet not consistent with patient preferences, and the lack of access to food and fluids. Malnutrition has been shown to be associated with functional decline at discharge from hospital; ${ }^{17}$

- Continence: The use of devices has been linked to deleterious effects such as pressure sores, recurrent urinary tract infections, depression, and functional dependency; ${ }^{18}$

- Length of stay (LOS): it was found a strong association between functional loss and length of stay. ${ }^{3}$ LOS was described to increase with the magnitude of prehospital functional decline, ${ }^{19}$ as well as to directly relate to HAD. ${ }^{20}$

In general, older age, dementia, functional decline, comorbidity and length of stay identify patients who are more difficult to discharge from hospitals and at higher risk of negative outcomes. ${ }^{3}$ 


\section{Functional recovery}

After hospital admission some patients do not recover to pre-hospital function, others can experience a worsening of function, but approximately $20 \%$ have significant functional improvement during hospital stay. $^{2}$ A full recovery to baseline level is typical of individuals with good premorbid characteristics (younger age, baseline independence, low comorbidity, limited preadmission decline). Anyway, functional recovery is much more frequent than functional decline in older patients. ${ }^{21}$

\section{Positive factors}

In the Progetto Dimissioni in Geriatria study, it was found that $59 \%$ of patients who experienced functional decline before hospitalization had some functional recovery during subsequent hospital stay, whereas only $1 \%$ worsened. ${ }^{21}$ In-hospital improvement was more frequent among patients with higher pre-morbid functional level and greater decline before hospitalization, indicating a resilient phenotype of geriatric patient who may benefit more from in-hospital interventions to recover function. ${ }^{21}$ In this study, the definition of functional improvement was a 5-point increase in Barthel Index, which is more sensitive to small changes in functional capacity than ADL scale.

\section{Negative factors}

A recent study found that cognitive and hearing impairment are significant predictors of functional decline during hospitalization. Moreover, severe cognitive impairment, visual impairment and weight loss before admission are associated with a reduced likelihood of functional improvement during hospital stay. 22

\section{Geriatric interventions to prevent HAD}

Geriatric interventions may be important to improve functional outcome during hospitalization:

- Early ambulation: Fisher and colleagues demonstrated that patients who increase their ambulation during the first $48 \mathrm{~h}$ after admission have reduced length of stay and are discharged approximately 2 days earlier than those who do not. This early ambulation is defined as walking at least 600 steps, that correspond to approximately $12 \mathrm{~min}$ of slow walking; ${ }^{23}$

- Acute Care of elders (ACE) units and Geriatric Evaluation and management
(GEM) units: ${ }^{24}$ ACE units have protocols for prevention and rehabilitation of disability; GEM units have a greater focus on rehabilitation than on HAD prevention, and treat patients after stabilization of their acute illnesses. ${ }^{24}$ The strongest evidence that redesigned care systems improve outcomes results from ACE units. A meta-analysis suggested that ACE units reduce the incidence of functional decline at discharge increasing the likelihood of going home and can reduce hospital length of stay; ${ }^{24}$

- Monitoring functional status over the course of hospitalization: the focus should start with the vision and attitudes of interdisciplinary teams suitable to geriatric needs; through changes in hospital processes affecting the physical and social environment; and end with continuity of care in the hospital-community interface targeted to improve functional status and quality of life. ${ }^{25}$ It is important to evaluate ADL function, cognition (by MiniCog test) and mobility (assess whether the patient can sit up in bed without assistance, stand and walk a few steps). Changes in functional status are important prognostic factors, predicting mortality and other health outcomes during and after hospitalization. $^{1}$

\section{Prognosis}

Hospitalization-associated disability is a high-rate phenomenon in the elderly and has a poor prognosis. A study with elderly patients who experienced HAD, showed that $41 \%$ died by 1 year, $29 \%$ remained disabled at 1 year, and only $30 \%$ returned to their baseline level of function. ${ }^{4}$ Information on patient prognosis to assess and track health status changes during hospitalization should be important to avoid unnecessary diagnostic procedures and inappropriate medical or surgical treatments in older people:

- The Walter Prognostic Index estimates the likelihood of 1-year mortality after hospitalization for older patients. The index uses 6 risk factors, all of which are easily available at hospital discharge (male sex, number of dependent ADLs at discharge, congestive heart failure, cancer, creatinine level higher than 3 $\mathrm{mg} / \mathrm{dL}$, low albumin level) and an additive point system; ${ }^{26}$

- The Multidimensional Prognostic Index (MPI) is the most accurate in predicting the risk of mortality. It is an index derived from Comprehensive Geriatric
Assessment (CGA) after hospital admission and based on six commonly used geriatric assessment scales exploring cognitive, functional, nutritional and clinical status, including also information about drugs taken and patient's social support. ${ }^{27}$ In older hospitalized patients, MPI predicts length of hospital stay, in-hospital mortality, and long-term mortality after hospital discharge..$^{27,28}$

\section{Conclusions}

Acute diseases and related hospitalizations are stressful and potential dangerous events in older persons that often leads to clinical complications, such as functional decline, prolonged length of stay, readmission, falls and mortality. Very old frail patients and those with preadmission functional limitation are at higher risk of complications. A new model of hospital care focused on functional status, including assessment on admission and throughout the hospital stay, promoting physical activity with early ambulation and planning for discharge home, is needed to reduce the incidence of HAD and to increase the likelihood of going home reducing hospital length of stay. About that, it was demonstrated that older patients who received CGA after admission to hospital to determine the medical, mental and functional problems at baseline, are more likely to survive and return home and are less likely to be admitted to a nursing home at up to a year after hospitalization. ${ }^{29}$ Therefore, availability of instruments to assess and track health status changes during hospitalization is very important to improve appropriate clinical management of older patients. Multidimensional Prognostic Index might be used to objectively track and monitor the clinical evolution of acutely ill geriatric patients admitted to the hospital with the aim of reducing length of hospital stay and facilitating a more efficient continuity of care and posthospital discharge management.

\section{References}

1. Covinsky KE, Pierluissi E, Johnston CB. Hospitalization-associated disability: "she was probably able to ambulate, but i'm not sure". JAMA 2011;306:1782-93.

2. Covinsky KE, Palmer RM, Fortinsky $\mathrm{RH}$, et al. Loss of independence in activities of daily living in older adults hospitalized with medical illnesses: 
increased vulnerability with age. J Am Geriatr Soc 2003;51:451-8.

3. Sleiman I, Rozzini R, Barbisoni P, et al. Functional trajectories during hospitalization: a prognostic sign for elderly patients. J Gerontol A Biol Sci Med Sci 2009;64:659-63.

4. Boyd CM, Landefeld CS, Counsell SR, et al. Recovery of activities of daily living in older adults after hospitalization for acute medical illness. J Am Geriatr Soc 2008;56:2171-9.

5. Brown CJ, Roth DL, Allman RM, et al. Trajectories of life-space mobility after hospitalization. Ann Intern Med 2009; 150:372-8.

6. Palleschi L, Fimognari FL, Pierantozzi A, et al. Acute functional decline before hospitalization in older patients. Geriatr Gerontol Int 2014;14:769-77.

7. Creditor MC. Hazards of hospitalization of the elderly. Ann Intern Med 1993;118:219-23.

8. Mudge AM, O'Rourke P, Denaro CP. Timing and risk factors for functional changes associated with medical hospitalization in older patients. J Gerontol A Biol Sci Med Sci 2010;65:866-72.

9. Fimognari FL, Pierantozzi A, De Alfieri $\mathrm{W}$, et al. The severity of acute illness and functional trajectories in hospitalized older medical patients. J Gerontol A Biol Sci Med Sci 2017;72:102-8.

10. El Solh A, Pineda L, Bouquin P, Mankowski C. Determinants of short and long term functional recovery after hospitalization for community-acquired pneumonia in the elderly: role of inflammatory markers. BMC Geriatr 2006;6:12.

11. Brown CJ, Redden DT, Flood KL, Allman RM. The underrecognized epidemic of low mobility during hospitalization of older adults. J Am Geriatr Soc 2009;57:1660-65.

12. Murphy EA. A key step for hospitalized elders: comment on "early ambulation and length of stay in older adults hospitalized for acute illness". Arch Intern Med 2011;171:268-9.

13. Steinman MA, Hanlon JT. Managing medications in clinically complex elders: "there's got to be a happy medium." JAMA 2010;304:1592-601.

14. Hammond T, Wilson A. Polypharmacy and falls in the elderly: a literature review. Nurs Midwifery Stud 2013;2: 171-5.

15. Ackroyd-Stolarz S, Bowles SK, Giffin L. Validating administrative data for the detection of adverse events in older hospitalized patients. Drug Healthc Patient Saf 2014;6:101-8.

16. Yoo JW, Kim SJ, Geng Y, et al. Quality and innovations for caring hospitalized older persons in the unites States. Aging Dis 2013;5:41-51.

17. Covinsky KE, Martin GE, Beyth RJ, et al. The relationship between clinical assessments of nutritional status and adverse outcomes in older hospitalized medical patients. J Am Geriatr Soc 1999; 47:532-8.

18. Zisberg A, Sinoff G, Gur-Yaish N, et al. In-hospital use of continence aids and new-onset urinary incontinence in adults aged 70 and older. J Am Geriatr Soc 2011;59:1099-104.

19. Rozzini R, Sabatini T, Cassinadri A, et al. Relationship between functional loss before hospital admission and mortality in elderly persons with medical illness. J Gerontol A Biol Sci Med Sci 2005;60:1180-3.

20. Zisberg A, Shadmi E, Gur-Yaish N, et al. Hospital-associated functional decline: the role of hospitalization processes beyond individual risk factors. J Am Geriatr Soc 2015;63:55-62.

21. Palleschi L, De Alfieri W, Salani B, et al. Functional recovery of elderly patients hospitalized in geriatric and general medicine units. The PROgetto DImissioni in GEriatria study. J Am Geriatr Soc 2011;59:193-9.

22. Gagliardi C, Corsonello A, Di Rosa M, et al. Pre-admission functional decline predicts functional improvement among older patients admitted to acute care hospital. J Gerontol A Biol Sci Med Sci 2017 [Epub ahead of print].

23. Fisher SR, Kuo YF, Graham JE, et al. Early ambulation and length of stay in older adults hospitalized for acute illness. Arch Intern Med 2010;170:1942-3.

24. Baztán JJ, Suárez-García FM, LópezArrieta J, et al. Effectiveness of acute geriatric units on functional decline, living at home, and case fatality among older patients admitted to hospital for acute medical disorders: meta-analysis. BMJ 2009;338:b50.

25. Admi H, Shadmi E, Baruch H, Zisberg A. From research to reality: minimizing the effects of hospitalization on older adults. Rambam Maimonides Med J 2015;6:e017.

26. Walter LC, Brand RJ, Counsell SR, et al. Development and validation of a prognostic index for 1-year mortality in older adults after hospitalization. JAMA 2001;285:2987-94.

27. Volpato S, Bazzano S, Fontana A, et al. Multidimensional Prognostic Index predicts mortality and length of stay during hospitalization in the older patients: a multicentre prospective study. J Gerontol A Biol Sci Med Sci 2015;70:325-31.

28. Pilotto A, Sancarlo D, Pellegrini F, et al. The Multidimensional Prognostic Index predicts in-hospital length of stay in older patients: a multicentre prospective study. Age Ageing 2016;45:90-6.

29. Ellis G, Gardner M, Tsiachristas A, et al. Comprehensive geriatric assessment for older adults admitted to hospital. Cochrane Database Syst Rev. 2017;9: CD006211. 